\title{
TRADICIÓN, REDES Y ORGANIZACIÓN EN LOS PUEBLOS DE LA CIUDAD DE MÉXICO Y LA DEFENSA DE SUS PANTEONES
}

\author{
TRADITION, NETWORKS AND ORGANIZATION IN THE TOWNS OF \\ THE CITY OF MEXICO AND THE DEFENSE OF ITS CEMETERIES
}

\author{
Oscar Cruz González*
}

\begin{abstract}
Tomando como estudio de caso una organización creada por pueblos de la Ciudad de México para mantener la administración de sus panteones, este trabajo busca mostrar algunos de los mecanismos que se pusieron en juego en la conformación de una organización que integraba a individuos y comunidades de distintas partes de la ciudad y que, por ello, tienen una historia diferente en su relación con ella. Se revisan aspectos como el papel que los panteones tienen en la vida comunitaria, las continuas relaciones que los pueblos mantienen entre sí y con las autoridades de la ciudad, las afinidades dadas por las fiestas y tradiciones y las relaciones que impone la división administrativa del territorio en la ciudad. Al mismo tiempo, se intenta enfatizar las formas en que este proceso puso a prueba las identificaciones por las que estas comunidades se han cohesionado social y políticamente, en este caso, la denominación pueblos en la ciudad.
\end{abstract}

Palabras claves: Ciudad de México, pueblos originarios, organización, redes, panteones.

Based on a case study about an organization formed by towns from Mexico City in order to keep management of their vaults, this work is intended to show some of the methods used to form an organization that joined individuals and communities from several places of the city; because of this fact, the relationship between these individuals and communities and their city is different. Several aspects are reviewed, such as: the role of cemeteries in community life; the constant relationships of the towns among themselves and with the authorities of the city; the affinities created by celebrations and traditions; and the relationships imposed by the administrative division within territories in the city. At the same time, emphasis is put on how this process tested the identities by which these communities have united socially and politically, in this case, the so called pueblos (towns) in the city.

Key words: México City, peoples, towns, organization, networks, cemeteries.

\section{Introducción}

En Ciudad de México aún persisten, y en ocasiones toman fuerza, comunidades que cuentan con muchos años asentadas en este territorio, algunas de ellas son anteriores a la conquista española. Estas localidades, ahora reconocidas como pueblos originarios ${ }^{1}$, son descendientes de diferentes grupos étnicos que poblaron la cuenca de México ${ }^{2}$ en el periodo mesoamericano y mantienen un vínculo con su pasado que se expresa en muchas de sus tradiciones y costumbres.

Producto del desarrollo histórico de la ciudad y sus distintos procesos de urbanización, en muchos pueblos su territorio ya no se diferencia claramente del resto de la ciudad ni cuentan con áreas naturales. De igual manera, en su interior no son homogéneos, sino que ahí conviven diferentes tipos de poblamientos $^{3}$. Aun así, en ellos se puede encontrar diversas formas organizativas por las que se relacionan en su interior, entre sí, y con la ciudad. Además de aspectos como la coexistencia de varios ciclos festivos que organizan temporal y espacialmente la vida comunitaria, la vinculación con el santo patrón y con la tierra, la presencia de sistemas de cargos y la definición (algunas veces solamente simbólica) de un territorio delimitado, se puede observar, ligados a estos elementos, la existencia de diferentes figuras de autoridad. Asimismo, en muchos de ellos se mantiene control de sus espacios, como son -y es el caso que se trata aquí- sus panteones.

En varios pueblos, aunque no en todos, sus panteones son administrados por los mismos habitantes por medio de diferentes figuras (como patronatos o comisiones, entre otras). Esto no ha sido del todo aceptado por las autoridades de la ciudad y otros actores políticos, quienes han buscado en distintas ocasiones que estos espacios sean dirigidos por personal del gobierno citadino.

En este texto trato el caso de un grupo de personas de varios pueblos de la ciudad que en el 2001 formaron una organización para confrontar

* Instituto Mora. México D.F., México. Correo electrónico: traos10@ hotmail.com 

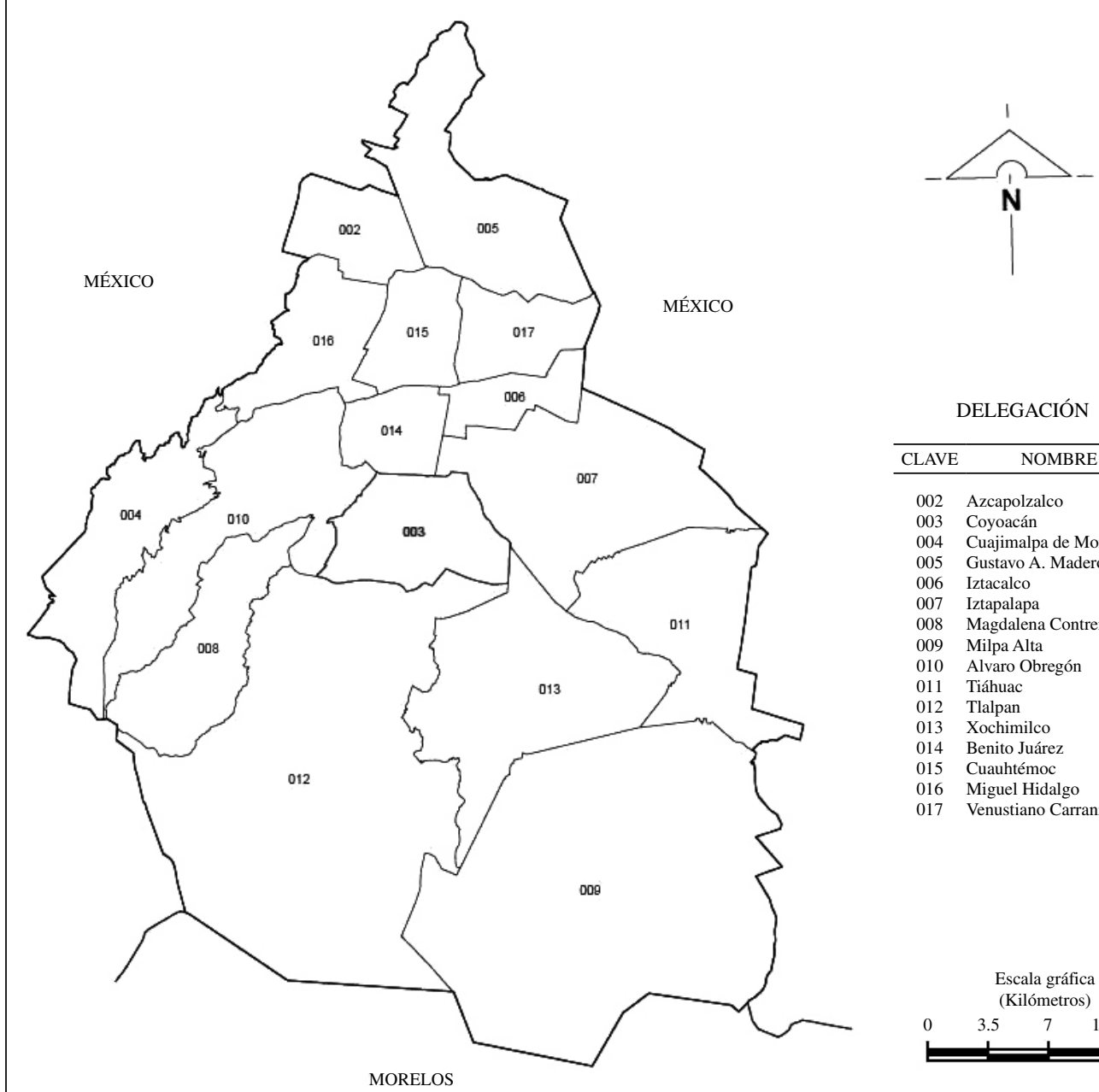

DELEGACIÓN

\begin{tabular}{cl}
\hline CLAVE & \multicolumn{1}{c}{ NOMBRE } \\
\hline 002 & Azcapolzalco \\
003 & Coyoacán \\
004 & Cuajimalpa de Morelos \\
005 & Gustavo A. Madero \\
006 & Iztacalco \\
007 & Iztapalapa \\
008 & Magdalena Contreras, La \\
009 & Milpa Alta \\
010 & Alvaro Obregón \\
011 & Tiáhuac \\
012 & Tlalpan \\
013 & Xochimilco \\
014 & Benito Juárez \\
015 & Cuauhtémoc \\
016 & Miguel Hidalgo \\
017 & Venustiano Carranza
\end{tabular}

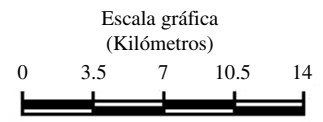

Figura 1. Ciudad de México. División por delegaciones.

Fuente: Instituto Nacional de Estadística y Geografía (INEGI).

la propuesta de ley de un diputado perteneciente al Partido Acción Nacional (PAN), que precisamente buscaba que los panteones de los pueblos fueran administrados por el gobierno de la ciudad. Quienes se enteraron de esta propuesta, habitantes de los pueblos de la delegación Coyoacán, en especial Los Reyes y San Francisco Culhuacán, buscaron a otros pueblos de la ciudad que serían también afectados por dicha ley y conformaron una asociación que finalmente, dos años después, se llamó Unión de Pueblos, Barrios y Colonias del Distrito Federal A.C. Esta Unión, después de movilizaciones y otras acciones, logró echar atrás esta propuesta de ley y otras que surgieron posteriormente.

Aquí se presenta el momento inicial de este proceso de lucha, en el que se buscó a otras comunidades y se les invitó a participar y conformar la organización. Además de mostrar una experiencia de organización que fue más allá de la concentración en la propia localidad, se hace hincapié en la importancia de los procesos iníciales en la formación de una organización social; ello desde un enfoque que observa este proceso como un problema de acción colectiva mediante la reconstrucción de la experiencia de los participantes.

La idea central es que en este primer momento se puede apreciar más claramente el funcionamiento de diversos mecanismos preexistentes para la construcción de una organización como esta, por ejemplo: la base comunitaria que organiza la vida local en cada pueblo y los vínculos entre pueblos, las afinidades dadas por las fiestas y otras tradiciones, y 
las relaciones que impone la división administrativa del territorio en la ciudad (Figura 1). Además, por medio del proceso de invitación y convencimiento se puede observar la complejidad y problemática de la conformación de una organización que buscó incluir grupos de diversas partes de la ciudad, con historias, necesidades e intereses particulares, aun cuando todos se cohesionaban en torno a una identidad particular, como en este caso puede ser la de pueblos originarios.

Para la argumentación, se parte con la presentación de la situación de los panteones en los pueblos de la ciudad, haciendo énfasis en la importancia que estos espacios tienen en la vida comunitaria en estas localidades. Después, sustentados en entrevistas a profundidad e investigación documental, se desarrollan los diferentes elementos observados en la organización mediante la discusión acerca de la importancia de los vínculos y redes previas -en el caso concreto expuesto aquí, de la consideración de la interrelación entre los vínculos festivos, religiosos y otros elementos simbólicos, las delimitaciones administrativas oficiales y los conocimientos de experiencias previas de organización social-. Igualmente, se trata la importancia de diversos mecanismos que intervienen en la acción colectiva, como la discusión y deliberación, entre otros, en la conformación de la organización.

\section{La importancia de los panteones para los pueblos}

Un aspecto fundamental de la conformación de la organización estudiada se encuentra en la relación con el tipo de problema que debían resolver, esto es, la administración de los cementerios, su gestión y su vínculo con el conjunto de su comunidad. Por ello es importante hablar, aunque sea de manera rápida, de los panteones de los pueblos, de cuál es su situación y, muy a grandes rasgos, de su importancia en la vida de estas localidades ${ }^{4}$.

De acuerdo con el Reglamento de Cementerios del Distrito Federal (1984) que se mantiene vigente (aunque con cambios), se pueden encontrar dos amplias clasificaciones de los cementerios existentes en la ciudad: los oficiales, también conocidos como civiles y los concesionados. Los primeros se pueden subdividir en tres tipos: a) Delegacionales, aquellos designados a atender gente de la misma delegación en la que se encuentra el cementerio; b) Generales (de los cuales dos se consideran "histórico generales"), que son en los que no importa la procedencia del difunto. Estos dos tipos están administrados por el gobierno del Distrito Federal (GDF) y son los que más superficie cubren, pues abarcan 505 ha. El tercer subtipo c) Vecinales, son los que se encuentran en los pueblos y en los que se atiende a difuntos que hubieran vivido en el "área vecinal correspondiente". El total de superficie que estos últimos abarcan es la menor, sumando únicamente 90 ha, lo que representa apenas menos del 10\% de la superficie total de los panteones en el Distrito Federal. El otro tipo son los panteones concesionados o privados cuya superficie es la segunda en extensión, con 230 ha (Yanes 2007).

La ciudad cuenta en total con 118 panteones que en conjunto cubren $825 \mathrm{ha}^{5}$. Aunque el mayor número de ellos cae en la denominación de "vecinales" encontrándose en territorio de los pueblos de la ciudad, el porcentaje de superficie que estos cubren es el más bajo, como se indicó. Se observa además que, dentro de la oferta del GDF, no se encuentra ni siquiera un panteón delegacional en cada una de las 16 demarcaciones. Adicionalmente, no en todos los pueblos existen panteones, al mismo tiempo que en un solo pueblo puede haber más de un panteón.

La normatividad en todos ellos se encuentra bajo la jurisdicción de "la autoridad sanitaria del Departamento del Distrito Federal (...) la Dirección General Jurídica y de Estudios Legislativos y de las Delegaciones del propio Departamento" (Departamento 1984) ${ }^{6}$. Por esto, se debe entender que los panteones que están en los pueblos no quedan fuera del marco jurídico-administrativo que rige a los demás, por lo que, fuera del aspecto de su administración y en lo referente a todos los demás puntos que marca el reglamento, deberán ajustarse a él. De esta manera, los cementerios que administran los habitantes originarios de los pueblos no dejan de estar ampliamente relacionados con el GDF, pero adquieren características propias que los convierten en espacios importantes dentro de los mismos pueblos.

Algunos panteones vecinales de la ciudad de los administrados por los mismos habitantes de los pueblos y en torno a los que giró la conformación y acción de la organización aquí estudiada, difieren en bastantes cuestiones administrativas de otros, sin embargo, es posible encontrar similitudes. Un importante punto coincidente en estos espacios es que quienes los administran son habitantes reconocidos como "originarios", siendo muy difícil y de hecho 
inexistente que la administración sea tomada por aquellos identificados como "avecindados". Esto reproduce la diferenciación originario/avecindado existente al interior de los pueblos y que se hace explícita y funciona de manera práctica en muchas otras cuestiones al interior de estas comunidades, como en las mayordomías. Esta división, aunque sólida, no es una norma inquebrantable y en función de los requerimientos de la situación puede flexibilizarse ${ }^{7}$.

Los "puestos" de los administradores, por lo demás, parecen ser siempre honoríficos, aspecto que los equipara con los "cargos" dentro de las mayordomías. En ambos casos, ser responsables es tomado como un servicio que se brinda al pueblo, a quien "le rinden cuentas" con asambleas periódicas. Además, los panteones, como bien es sabido, son espacios de culto y de diversas tradiciones, que relacionan a los habitantes de los pueblos con sus antepasados y, por ello, son importantes lugares de la memoria colectiva local.

En cuanto a aquellos aspectos en los que difieren, se puede mencionar el hecho de que su origen está en procesos como la donación o compra en común del terreno, la utilización de tierras comunales o ejidales o por una combinación de estos aspectos. Esto les otorga un sentido de "pertenencia" sobre este espacio y es, por ello, su panteón. También varían las formas de organización que adoptan y los vínculos de la comunidad con ellos, de tal manera que pueden estar administrados por patronatos, comisiones, asociaciones civiles y representantes, entre otras. Además, debido a que la mayoría de los administradores de los panteones son elegidos en asambleas, ellos consideran que su responsabilidad principal está con los usuarios-habitantes y no con las autoridades gubernamentales. Hay entonces un estrecho vínculo, de servicio y de confianza, entre estas figuras y los habitantes del pueblo.

También varía entre pueblos la forma cómo se asignan los espacios (las fosas): a perpetuidad o temporales, decisión que depende mucho de la saturación del espacio y de la incorporación de nuevos "usuarios" que no reservaron un lugar anteriormente, por el que pudieran respetarles la perpetuidad, además de los cambios decididos en asamblea. La existencia de reglamentos de funcionamiento del panteón es otro punto donde hay diferencias. Puede ser que se tenga un documento explícito o que se conozcan algunas normas de manera oral. En cualquier caso existe un grado de flexibilidad para su aplicación, según lo consideren los encargados. En materia de cobros o gratuidad del servicio hay también divergencias en cuanto a la temporalidad y el monto. Debido a este manejo de los recursos económicos se puede observar en estos cementerios algún grado de autonomía frente a las disposiciones del GDF. Al mismo tiempo, en la mayoría de ellos los futuros beneficiarios del servicio deben participar periódicamente en la limpieza, pintura, desyerbado y otras actividades.

En conjunto, estos aspectos revelan formas de organizarse comunitariamente, de extender la relación con lo civil hacia lugares en donde la gestión del pueblo y las reglas de la autoridad gubernamental se entrecruzan, a veces fácil, a veces conflictivamente. Muestran también la relación cercana entre el conjunto del pueblo y aquellas personas que cumplen ciertos servicios en la comunidad, dentro de un aspecto más participativo. Expresan el papel de estos lugares como espacios de identidad de sus pueblos mediante el control que la comunidad tiene en sus procesos y la relación con otras formas de organización local, como las mayordomías. Asimismo, el papel que se le reconoce a los panteones en la vida de los pueblos tiene un carácter dinámico, ya que así como en muchos se mantienen bajo el control de los mismos habitantes, en otros se ha perdido y en algunos más el interés por ellos es reciente.

\section{Las propuestas de modificación}

Vistos así los panteones de los pueblos, no es de extrañar que una iniciativa de ley que propusiera cambiar la administración no pasara inadvertida y fuera motivo suficientemente importante para organizarse y frenarla. Lo que planteaba la propuesta de octubre de 2001, impulsada por el diputado del PAN, Víctor Hugo Gutiérrez Yáñez, era, en términos generales, la regulación del uso y manejo de cementerios en el conjunto de la ciudad, incluyendo a los vecinales ${ }^{8}$.

Esta iniciativa de ley se centraba en dos puntos. El primero era modificar el reglamento para que los panteones en la ciudad fueran clasificados en dos grandes ámbitos: oficiales y concesionados; la idea era que en los oficiales se eliminaran los distintos subtipos y entonces los vecinales desaparecerían como tales. El segundo era que, una vez hecho lo anterior, los panteones antes vecinales pasarían a ser administrados por el GDF como los demás. 
Estas acciones tendrían efecto, además, sobre ciertas reglas tácitas y algunas formalizadas que operan en los panteones de los pueblos como que allí son enterrados únicamente quienes vivieron en la comunidad y fueran reconocidos como originarios. Igualmente, como consecuencia, también se eliminaría la modalidad de fosas a perpetuidad, que oficialmente había sido abolido en $1974^{9}$, pero que en muchos panteones de los pueblos se mantiene vigente. En una mirada general, esto fue interpretado no solamente como un ataque a un espacio particular del pueblo, sino que, por las características que ya se mencionó, se consideró como un atentado contra sus usos y costumbres en su conjunto.

Un aspecto importante al respecto es que los pueblos no parecen estar en desacuerdo con buscar formas de solucionar problemas relacionados con sus panteones, como la saturación. Por ejemplo, uno de los proyectos con los que inició el contacto con el gobierno por medio de la Unión, fue para pedir un permiso para hacer exhumaciones. La principal diferencia en este caso es que esta decisión había sido tomada en asamblea en el pueblo, si bien la iniciativa partía del análisis efectuado por los miembros de la comisión del panteón. Otro asunto fue que, aunque en un número muy pequeño y $\sin$ dejar de ser un asunto importante en la diferenciación de derechos al interior de los pueblos, en los panteones vecinales no solamente se entierran personas consideradas como originarias, sino que, de acuerdo con lo acordado en asambleas y de la disponibilidad de espacio, se puede sepultar a los avecindados. Finalmente, la decisión de ser tan restrictivos con quien se entierra en estos espacios está mediada por un sentido de pertenencia que se expresa en el conocimiento mutuo de los habitantes del pueblo, así como de sus familias, de tal manera que el panteón funciona como un vínculo entre ellos, toda vez que se está al pendiente de él constantemente, en reuniones semanales (o como lo designe cada administración); que se refuerza en relaciones informales en la vida cotidiana del pueblo y que por conducto de ellas se convierte -al panteón- en un espacio de reunión (como también de disputa) para el conjunto de los habitantes.

Esta es la problemática que enfrentaban los administradores de los panteones y que se puede expresar en tres puntos: 1) el ámbito de los panteones no solamente significaba el mantenimiento del control de un espacio dentro del pueblo, sino que tiene significados propios que se arraigan en la historia, vida y sentido de aquellas comunidades, 2) era un ataque del gobierno hacia una de las pocas cosas que habían podido recuperar de entre todo lo que la conformación histórica de la ciudad les había quitado y; 3) por estas características, era un problema ante las autoridades gubernamentales propiamente, que al parecer no involucraba otros actores.

\section{La conformación de la Unión. Redes, micromovilización y organización}

Los inicios de la organización que se conformó para enfrentar esa propuesta de cambio son curiosos y muestran la importancia de la conjunción del azar y la preexistencia de vínculos comunitarios. En efecto, de acuerdo con los entrevistados, la forma como se enteraron que la existencia de la propuesta de ley del 2001 para la modificación del reglamento de cementerios fue por un descuido de parte de funcionarios del GDF. Acerca de aquel evento, sin embargo, no hay una única narración y se encuentran al menos tres diferentes versiones.

Aquí no es muy importante centrarse en cada versión y más bien se dice que las tres tienen elementos comunes. Aunque es posible hacer algunas anotaciones. A pesar de que en esas versiones se refieren años distintos, 2001 y 2002 (aunque realmente la diferencia es de meses), no se puede asegurar que alguna sea falsa. Esto implica dos cosas. Primero, en este caso, por parte de los participantes en la organización no hay una preocupación mayor por conocer si alguna versión es copia de la otra o si algún grupo modificó los hechos para ser protagonista. En la organización no existe una monolítica narración de su historia, las diferentes versiones pueden tener cabida en la medida en que además aportan el elemento básico: el descubrimiento vía el azar; fuera de ello, los detalles no les preocupan mucho. Segundo, metodológicamente no se buscó fehacientemente conocer cuál versión era la correcta; en este nivel no interesa saberlo (amén de que es muy complicado averiguarlo), pues la reconstrucción del proceso, por estar ligado a la construcción identitaria que se llevó a cabo, está más fundada en las narrativas que en la obtención de pruebas que pudieran desmentir a alguno de los entrevistados en aras de "encontrar la verdad". Lo importante es cómo la recuerdan y la narran y los efectos que eso tiene o no para su práctica posterior y las acciones de la Unión. De la misma manera, pensar esas diferentes versiones como la reconstrucción de la 
experiencia personal, que por lo tanto no puede ser "falsa" ni "verdadera", ayuda a relacionar el plano individual con el de la organización.

También, parece que este señalamiento del azar solo sirve como el detonador de una situación que, según entrevistas, había ya comenzado a articularse en vista de algunos otros procesos de conocimiento entre distintos patronatos de los panteones. Ejemplo de ello es uno que refiere a la construcción de un primer vínculo con otros encargados de panteones a partir de visitar a los responsables de ellos para saber si en todos se estaba realizando un cobro de impuesto predial que se hacía en el panteón de San Francisco Culhuacán y que les resultaba extraño ${ }^{10}$, situación que además de vincular a distintos panteones los comenzó a acostumbrar a dirigirse a las autoridades. Mediante procesos como este se puede observar que en la conformación de la organización, en la decisión de su creación, confluyen el azar y un trabajo previo de reconocimiento. Por ello puede decirse que el azar influyó solamente en la medida en que los interesados (los habitantes de los pueblos), por las continuas relaciones que tenían con las autoridades, a las que se buscaba, se encontraban en el lugar adecuado en el momento justo.

La cuestión más importante aquí es que en las tres versiones al enterarse acerca de la existencia de la propuesta de ley, la respuesta de los pueblos fue, primero, difundir su existencia y contenido, invitar a otros pueblos a unirse para oponerse a ella y no dejar que se aprobara. El proceso no era fácil y se requería convencer a los encargados de los panteones en otros pueblos, lo que se logró por medio de reuniones con ellos. En este proceso intervienen los vínculos previos, conocimientos $\mathrm{y}$ experiencias que se han forjado entre pueblos.

Teóricamente se ha tratado este tema desde diversas perspectivas. En la literatura sociológica relativa a movimientos sociales se reconoce que una organización o movimiento no surge de manera espontánea, sino que para ello se necesitan diversas condiciones que canalicen los sentimientos de agravio e injusticia que experimenten individuos o colectivos y sean vinculables con organizaciones y otros agrupamientos. De la misma manera, se opina que la existencia de un cierto grado de organización es necesario para que una acción colectiva más compleja, como un movimiento social, cohesione a los participantes y pueda perdurar.

En el momento de creación de una organización como la aquí tratada, que no se fundó con el objetivo principal de disputar recursos escasos en competencia con otras organizaciones y por ello está más cercana a la acción de los movimientos sociales, los elementos culturales propios de los grupos que la construyen son muy importantes. Para Alberto Melucci, por ejemplo, la existencia de lo que llamó redes sumergidas (Melucci 1994, 1999), es decir, aquellas relaciones que se establecen ya sea por la participación en la comunidad o de experiencias pasadas y que se mantienen latentes, es decisiva para la conformación de un movimiento social, pues abonan a la construcción de la identidad colectiva que adoptará el movimiento en la medida en que, al actuar durante el periodo de latencia, sientan las bases de la vinculación de los individuos participantes en las organizaciones y el movimiento con sus entornos inmediatos y su vida cotidiana.

Estos vínculos se potencian en los momentos de movilización y de organización, ya sea informal o formal. Este anclaje en la vida cotidiana influye también en la forma organizativa y el grado de formalización que adquiera. En efecto, la forma organizacional que un colectivo adopte no es solamente un aspecto instrumental, sino que puede ser también un fin en sí mismo en la medida en que los grupos organizados buscan fundarse en sus ideas, creencias, tradiciones e ideologías para construir sus organizaciones y, así, utilizar sus recursos materiales y simbólicos (Melucci 1999; Escobar et al. 2001). De esta manera, la base cultural dentro de una organización, vista en buena medida en redes cotidianas, cumple dos funciones: por un lado la vincula (a la organización) con la comunidad y, por otro, delinea la forma organizacional (rigidez o formalización de las normas internas, membresía, jerarquías y demás) que se adquiera.

También es por este elemento cultural que los grupos organizados conforman una identidad donde no todo es negociable. Aunque aceptemos que hay un elemento instrumental en la construcción de la identidad colectiva, en particular referido a la conformación y uso de un determinado discurso, esto no implica que la identidad solo se constituya instrumentalmente, sino que incluye también, y en una buena medida es su ingrediente principal, los reclamos que se fundan en un modo de vida determinado; esto hace que haya elementos en la identidad colectiva que, por fundarse en las experiencias de vida de los participantes y sus comunidades, son más difíciles de ser cooptados por los poderes a los que hacen frente. 
Sin embargo, tampoco debe pensarse que la vinculación comunitaria determina directamente la identidad asumida y la forma organizacional adoptada. Estas se construyen de una manera relacional con reuniones, disputas, deliberaciones y negociaciones que los grupos hacen involucrando, además, los procesos políticos que se dan en el contexto de las acciones. En este sentido, otro elemento importante es la idea del territorio, como un espacio apropiado y definido afectiva, simbólica y materialmente, y que es incorporado a la construcción identitaria de los sujetos. Este territorio también se construye y redefine en interacción con otros grupos.

Todo ello presupone que la acción no está definida de antemano. Aunque existan varios repertorios de protesta, es decir, formas más o menos tradicionales o afines a un grupo particular (marchas, negociaciones, acciones directas, presión a delegados y demás), las acciones se darán, en buena medida, por la creatividad de los miembros, a partir tanto de definiciones estratégicas de la situación (Jaspers 2004) dentro de una determinada arena política como de sus orientaciones culturales propias.

Así se puede pensar también la conformación de una determinada forma organizativa fundada en orientaciones culturales. Como se mencionó, esto implica la elección no solo de la manera cómo se construye su estructura, por ejemplo las tomas de decisiones, la jerarquización al interior y el establecimiento de relaciones horizontales y verticales, sino también en su afiliación a determinados discursos y posiciones políticas e ideológicas presentes en, por ejemplo, la idea de la conformación de una ONG o A. C., entre otras (ver: Canto 2004). Ser una Asociación Civil, por ejemplo, en el caso aquí tratado, tenía varias ventajas sobre otras formas organizativas: por una parte, y este era el elemento central, permitía poseer una figura jurídica para poder tener representatividad; por otra, al ser la asamblea el órgano principal de una A. C., por encima de la mesa directiva, quedaba en sintonía con las prácticas de toma de decisiones de sus comunidades. Así, aunado a que en sus discursos no hay referencias a conceptos como el de sociedad civil, la necesidad de constituirse instrumentalmente en una organización más formal se unía con la cercanía que en ciertos puntos ofrecía la forma de asociación civil.

Desde este punto de vista, la organización se encuentra en un nivel intermedio entre lo individual y lo estructural, vinculándolos. En ella los intereses individuales, que no solo se entienden como aquellos objetivos inmediatos y generalmente materiales que los participantes buscan al colaborar en una organización, sino que esta les provee también de incentivos de solidaridad y valorativos (Melucci 1996) se encuentran con la acción del conjunto que no es la suma de las acciones individuales, sino que es posible identificar como una acción distinta, la de un actor colectivo. Para reducir la complejidad que esto representa, entre estos niveles se puede dar una relación de "autonomía". Matilde Luna y José Luis Velasco (2010), por ejemplo, llaman "doble autonomía" a la situación en la que, dentro de una asociación, entre los participantes individuales (que pueden ser individuos o grupos) y el conjunto, o sea la asociación como un todo, existe un cierto grado de libertad. Aquí estos dos niveles (las partes y el todo) son diferenciables, es decir, los integrantes pueden tomar diferentes decisiones y son identificables en sí mismos, y la asociación más amplia también es vista como un actor con derecho propio.

Creo que este es un marco teórico adecuado para poder pensar lo que ocurrió en el caso de esta organización, pues vincula la conformación de organizaciones no solamente a los interesados por algún problema en común, sino que resalta la importancia de los contextos cotidianos y los múltiples entretejimientos entre actores que se dan en ellos, de los factores culturales y de la identidad que se construye. Asimismo, aunque es importante reconocer la utilidad (y necesidad) de recursos materiales para la organización y movilización, no se deben dejar de lado los de carácter simbólico ${ }^{11}$.

Sin embargo, este proceso no es tan sencillo. La preexistencia de esas relaciones latentes no se convierte en alguna organización (o movimiento) de manera lineal. Como se menciona anteriormente, este es un proceso marcado por debates, diálogos, acciones de convencimiento y negociación e implica que en cada una de estas acciones existan mecanismos propios, como la deliberación, la relación de fuerzas, la argumentación y pruebas, entre otros. En el caso aquí estudiado, esto se da de una manera importante al inicio de la organización, en el proceso que se puede llamar de "micromovilización"12, que se refiere al contacto, convencimiento y reclutamiento de nuevos miembros y que se puede ampliar a la búsqueda de los posibles apoyos, aliados o simpatizantes. Así, a partir de su invitación el conflicto se 
hace público y se van construyendo las posiciones/ identidades involucradas.

Resumiendo, en la conformación de organizaciones y en especial de organizaciones complejas como la aquí tratada, que está compuesta por otros grupos e individuos, la preexistencia de redes, de vínculos entre distintos grupos y que están asentadas en la vida cotidiana de las comunidades es un factor clave para la forma organizacional que se adopte. También tiene efectos en los procesos de búsqueda de participantes y aliados que, al recurrir a esas redes, construye en el proceso una identidad colectiva particular por la que, y junto a los diversos incentivos que la organización brinda a los participantes, se buscará lograr los objetivos propuestos.

Volviendo al caso aquí tratado, la primera acción al conocerse la propuesta de ley fue la comunicación con otros pueblos, pero la particularidad fue que el primer acercamiento lo buscaron entre pueblos de la misma delegación. En efecto, aun en el caso de San Francisco Culhuacán, que en una de las versiones son quienes se enteraron de la existencia de dicha ley, se acudió más bien a los panteones de otros pueblos de la delegación Coyoacán que al otro panteón que existe en el mismo pueblo, pero se encuentra en la delegación Iztapalapa. La delimitación territorial al respecto es interesante, porque señala afinidades, relaciones formales e informales por fiestas y otras celebraciones, pero también muestra la importancia que para tratar asuntos con autoridades de distintos niveles de gobierno en la ciudad ha obtenido el plano delegacional, tanto por ser la forma de territorialización administrativa que funciona en la ciudad como por el proceso de elección de los delegados desde el 2000 y las relaciones entre estos y los habitantes de las delegaciones.

Después de este proceso de comunicación entre los pueblos de la delegación Coyoacán (Los Reyes, San Francisco Culhuacán, Santa Úrsula Coapa, San Pablo Tepetlapa y La Candelaria, con una buena respuesta de ellos, interesándose en la problemática), siguió la invitación a los pueblos de otras delegaciones, pero para ello ya se había conformado, aunque no de manera explícita, un "centro" desde donde se concentrarían las futuras acciones: Los Reyes Coyoacán.

El primer lugar fuera de su delegación al que acudió el grupo que supo del proyecto de ley fue a Xochimilco, pero en esta primera visita solo contaban con el conocimiento de la propuesta de ley, no la tenían físicamente. La falta de ella y el mínimo conocimiento de lo que refería hicieron que los habitantes de Xochimilco no se interesaran en un inicio en ayudarlos a hacer frente a tal proyecto, pues al no saber bien qué decía la iniciativa suponían que a ellos no les afectaría y que era un asunto únicamente de la delegación Coyoacán. Igualmente, el rechazo inicial de Xochimilco estaba fundado en, por un lado, su creencia de que a ellos, por ser un pueblo reconocido como patrimonio de la humanidad, no se les afectaría de esa manera (Romero 2010) y, por otro lado, en la relación más o menos funcional que, sentían, existía por medio de la figura del Coordinador de Enlace Territorial (CET) ${ }^{13}$ como vínculos entre las comunidades y la delegación, que a diferencia de los pueblos de Coyoacán aquí sí operaba.

Ante esta negativa, y reconociendo lo complicado que sería conseguir apoyo si no se contaba con el conocimiento claro de lo que implicaba una reforma como la propuesta, decidieron "solicitar información en la oficina de panteones de la Consejería Jurídica del GDF. La respuesta que obtuvieron fue que no existía ninguna iniciativa de ley" (Romero, 2010). Sin embargo, debido a un acto de suerte, logran "encontrarse" con el documento.

Con el impreso en su poder, pudieron estudiarlo mejor, saber qué implicaba y, más que todo, probar a otros pueblos que no era un asunto limitado a una delegación, sino que les afectaba mientras estuviera dirigido al conjunto de cementerios en la ciudad. De esta manera, los representantes de los panteones de Coyoacán regresaron a Xochimilco y esta vez, con la iniciativa en mano, sí aceptaron participar, difundirlo e invitar a otros pueblos. La insistencia de ir a Xochimilco se explica por los vínculos que mantenían con ellos y en especial por el reconocimiento de que ahí vivían unas personas que tenían experiencia tratando asuntos similares (de defensa del territorio) y podían asesorarlos. También era importante que en Xochimilco se reunían desde hace tiempo pueblos de las delegaciones en donde se eligen CET: el mismo Xochimilco, Tlalpan, Tláhuac y Milpa Alta ${ }^{14}$.

El proceso de difusión inicial estuvo sustentado en dos estrategias. Por una parte la utilización de redes establecidas previamente con otros pueblos, a partir tanto de cuestiones de los panteones como de diversas celebraciones religiosas, ya que la mayoría de quienes participan en la administración de los panteones han estado también en otros cargos al interior de sus pueblos, ya sea como mayordomos, en las comisiones y demás, o conocen a quienes 
lo son, de tal manera que, también por esta vía, han establecido relaciones con otros pueblos; además de que refleja el potencial de los sistemas de cargos en el establecimiento de relaciones de carácter político. Por otro lado, como en el caso de Xochimilco, se utilizó una especie de "bola de nieve" en la que cada pueblo contactado se comunicaba con otros con quienes tuvieran relaciones. Pero el trabajo de invitación y convencimiento siempre fue cara a cara con visitas a los distintos pueblos y en muchas ocasiones se desarrolló por medio de debates.

En esos debates se deliberaba tanto acerca de las implicaciones que para los pueblos tendría esta iniciativa como respecto de las acciones a tomar, ya que la urgencia de detener la propuesta de ley apremiaba a apresurar el proceso e ir definiendo acciones sobre la marcha. En reuniones constantes, llevadas a cabo en los espacios de los pueblos, en ocasiones en sus panteones y en las que a manera de fiesta muchas veces al terminar se invitaba a una comida, también se iba definiendo la estructura organizativa a tomar y, más adelante, la decisión de convertirse en una Asociación Civil ante la necesidad de conformarse como persona jurídica colectiva (moral).

Este tipo de "redes" existentes entre los pueblos también estaban preestablecidas entre los encargados de los panteones por procesos recientes al conocimiento de la iniciativa de ley. Mediante acciones como solicitar información acerca de cobros o para algunos cambios en la forma de administrar su panteón, se construyeron vínculos con otros encargados de panteones, pero también con ciertas instancias gubernamentales, encargadas de atender la problemática en estos espacios. Esto les dio no solo el conocimiento de quienes eran los responsables en tal rubro, sino también cómo proceder en ellos, adquiriendo el lenguaje y los códigos necesarios.

Con el reconocimiento de que se necesitaba construir formas en las que se pudiera hacer frente a la propuesta de ley, los representantes de los panteones iniciaron el proceso de organización con una estructura más bien informal en el sentido de que no existía ningún documento en el que se plasmaran normas internas ni existía un registro legal. Esto no impedía que utilizaran un nombre, se movilizaran y entablaran diálogos y exigencias a las autoridades; de hecho, las bases que se asentaron en esta forma de organizarse estuvieron presentes aun cuando se formalizó la Asociación Civil.
Desde el inicio de sus acciones, la estrategia fue dirigir oficios a diputados de la Asamblea Legislativa del Distrito Federal (ALDF) para que fuera rechazada la propuesta de ley. De acuerdo con algunos de estos documentos, fechados en diciembre de 2001 y enero de 2002, la organización en estos se hizo llamar Unión de Pueblos del Distrito Federal y a quienes representaba era a los pueblos de las delegaciones Coyoacán, Tláhuac, Iztapalapa y Magdalena Contreras. En otro oficio, fechado el 17 de abril de 2002, firmaron como Unión de Pueblos y Barrios del Distrito Federal. El 8 de mayo del mismo año también hicieron otro oficio con el mismo nombre; así que a partir de este momento se la puede identificar como Unión. En estos últimos los pueblos representados eran los mismos que en los anteriores. Además, en estos documentos hacían público el lugar en el que ubicaban su domicilio legal, en el pueblo de Los Reyes Coyoacán.

Se puede observar entonces que para diciembre de 2001, el mismo año en el que se enteraron de la existencia de la propuesta de ley, ya habían logrado adherir a pueblos de distintas delegaciones. Al mismo tiempo se consideraban ya un grupo que podía ser reconocido con un nombre que hacía referencia a sí mismos como pueblos, aunque no se le adhería el adjetivo de originario (realmente este adjetivo nunca se utilizó, aunque sí existía la idea de considerarse como tales ${ }^{15}$ ). Dichos nombres son bastante generales, como pensados para ser incluyentes, sin que algunos integrantes pudieran sentir que a ellos pertenece la organización, aunque había un sentido claro de quiénes habían sido los iniciadores y los "derechos" que ello les confería, ligados en particular a la presidencia y la mesa directiva.

La composición de la Unión, es decir, cuántos representantes de panteones la integraron en sus diferentes momentos y cuánta gente se juntaba en las reuniones, no es bien conocida. Por ejemplo, existe una discrepancia en cuanto a la cantidad de pueblos que, según algunos entrevistados, se sumaron a los esfuerzos de la organización por delegaciones $\mathrm{y}$ aquellas delegaciones en las que existen panteones vecinales. Un entrevistado recuerda que solamente alrededor de dos delegaciones no participaron ${ }^{16}$, pero según Pablo Yanes los panteones vecinales están ausentes en las delegaciones Cuauhtémoc, Benito Juárez, Miguel Hidalgo, Venustiano Carranza e Iztacalco $^{17}$ (Yanes 2007:222). Debido a que sabemos que no participaron quienes no fueran representantes de un panteón y no existen registros en manifiestos u 
oficios que mencionen a estas últimas delegaciones (las mencionadas por Yanes) es plausible pensar que no había ningún integrante de ellas en la Unión. En delegaciones como Gustavo A. Madero, según cuenta un entrevistado, no participaron porque ahí la delegación controla los panteones, aun cuando los habitantes de los pueblos no permitan que se entierre a quienes no vivían en el pueblo ${ }^{18}$.

Quizá la fuente más confiable para determinar esto sea un directorio interno más acabado, en donde se podía encontrar gente de las siguientes delegaciones y pueblos que participaban en la Unión: Magdalena Contreras (Magdalena Contreras, San Bernabé Ocotepec), Azcapotzalco (San Andrés), Xochimilco (Santiago Tepalcatlalpan, San Mateo Xalpa, Santa Cruz Xochitepec), Álvaro Obregón (sin referencia clara a un pueblo en particular), Coyoacán (La Candelaria, Los Reyes, San Francisco Culhuacán, Santa Úrsula Coapa, San Pablo Tepetlapa, además de la colonia Santo Domingo), Tlalpan (San Miguel Ajusco), Iztapalapa (Culhuacán), Tláhuac (San Francisco Tlaltenco, Santiago Zapotitlán) y Milpa Alta (San Jerónimo Miacatlán). Además, según entrevistas, en las sesiones de la Unión acudían ejidatarios y distintos mayordomos.

Ahora bien, es necesario explicar una parte del nombre de la Unión, aquella que incluye a las colonias. Como vemos en el listado de delegaciones y pueblos se incluye la colonia Santo Domingo, esta fue la única que tenía presencia en la Unión. Su inclusión estaba definida no por su condición de colonia, sino por su anterior pertenencia, antes de la invasión de la noche del 3 de septiembre de 1971, como parte de los terrenos del pueblo de Los Reyes. Después de aquella invasión y durante las distintas etapas de la formación de la colonia algunos habitantes del pueblo se trasladaron a vivir en ella, pero mantuvieron muchos vínculos con el pueblo ${ }^{19}$. Son estos habitantes, con derecho a ser enterrados en el panteón del pueblo, los que participaban como parte de las colonias.

Muchas de las estructuras organizativas y formas de acción que desplegó la organización se dibujaron desde el periodo inicial. En efecto, si se observa el desarrollo de sus acciones se puede ver que estas no variaron, sino fueron los mismos canales: comunicados a las autoridades, oficios, búsqueda de diálogo, marchas, comunicación y desplegados al interior de los pueblos y en medios de comunicación (periódicos, que más que desplegados fueron publicados en secciones como "El correo ilustrado" de La Jornada). Medidas y acciones que casi no variaron con el registro como Asociación Civil.

Además de lo ya señalado acerca de los oficios dirigidos a legisladores dentro de la ALDF, se puede mencionar la importancia del carácter de la primera marcha a la ALDF desde el metro San Antonio Abad, efectuada en el 2002. De acuerdo con los entrevistados, desde un inicio se definió que las acciones a tomar se evaluarían dependiendo de la situación, pero se llegaría hasta donde se tuviera que llegar para solucionar sus problemas. En este sentido, la marcha se efectuó después de no recibir respuesta favorable por parte de los legisladores ni otras autoridades del GDF. Esta primera acción se caracterizó por hacer uso de un conjunto de elementos "festivos" (en el sentido de que animaban la marcha), que llamaron la atención de periodistas y fotógrafos y son, ahora, recordados con risas por parte de los entrevistados. Pero eran realmente elementos propios de los rituales funerarios. Estaba así una banda de música (como las que acompañan a un difunto por las calles de los pueblos) una corona de flores y un ataúd, por ejemplo. Este es el tono que tuvieron las posteriores movilizaciones. Los entrevistados ahora recuerdan aquellas expresiones y las ligan con la tradición y forma de ver la muerte que se tiene en los pueblos de raíz mesoamericana ${ }^{20}$.

Por tanto, en los discursos públicos como en las expresiones vertidas durante las movilizaciones se comenzó a formar una identidad del grupo y se definió así a quienes representaría esta organización, los integrantes, que participarían como representantes de los panteones más que a título individual. Al mismo tiempo se identificó al gobierno de la ciudad como la única contraparte en este conflicto. Por consiguiente, se delimitó el campo de actores reduciéndolos a estos dos, sin involucrar a otros, como pudieran haber sido otras organizaciones sociales y actores, lo que también significó que no existían intermediarios entre las dos partes. Tal relación, además, no era completamente antagónica, sino que buscaba diferentes canales para solucionar las problemáticas existentes, si se pudiese de una manera que no representase un conflicto mayor. Aun así, como dice un entrevistado, con el paso del tiempo los integrantes consideraban que "sí se llegó a tener esa calidad de oposición frente al gobierno"; "sí, definitivamente si nos vio [el GDF] como una oposición muy sólida"21.

Finalmente, en este periodo se fundaron también algunas de sus características organizativas. Como 
representantes de sus panteones, los integrantes de la Unión debían consultar con los otros encargados de estos espacios en sus pueblos acerca de las disposiciones que se adoptaban en la organización. Asimismo, recaía en ellos comunicar en sus pueblos los avances y nuevas acciones que la Unión tomara, de tal manera que hasta cierto punto se convertían en un vínculo no solamente entre la organización y los cementerios, sino también con el pueblo en su conjunto.

A pesar de estas características, la Unión tenía limitaciones para conseguir sus fines (en este momento la detención de la propuesta de ley): no tenían personalidad jurídica. En efecto, al no plantear una relación completamente antagónica con el GDF la Unión había optado por conducirse dentro de los canales "oficiales", pero para jugar bien se necesitaba ser reconocidos como sujeto jurídico, lo que les permitiría, entre otras cosas, conseguir amparos o entablar juicios como persona moral. Este hecho representa uno de los primeros enfrentamientos con la realidad institucional en la que se tenían que ajustar. De esta manera, la decisión de conformarse como Asociación Civil era la solución, además, en el pueblo de Los Reyes ya se había experimentado esta misma figura con relación a sus mayordomías ${ }^{22}$.

\section{Conclusiones}

La existencia de esta Unión no es algo casual, ni fácil. Deriva, por un lado, de un momento en el que los pueblos de la ciudad han transcurrido por distintas autodefiniciones en el conjunto de la ciudad, como una parte central de ella y politizándose en torno de autoadscripciones como la de "originarios". También es un momento en el que, resultado de esos mismos reconocimientos y movilizaciones, se comenzaban a crear espacios de participación y de integración entre pueblos que iban más allá de (aunque se sostenían en) los vínculos que ellos mismos han creado para realizar sus fiestas, peregrinaciones, correspondencias y demás aspectos culturales, con miras a tener un mayor poder de decisión en la política de la ciudad. Además, la conformación de esta Unión también expresó las distintas dificultades de desarrollar acciones colectivas aun entre sujetos colectivos que se reconocen como similares y mantienen un conjunto de vínculos previos.

El tema aquí expuesto refleja, o si se quiere, refuerza, la visión de las complejas relaciones que se establecen en el proceso de conformación de una acción colectiva destinada a involucrar a comunidades pertenecientes a distintas partes de la ciudad, que han tenido diferentes experiencias con ella y mantienen diversos intereses y necesidades. La adscripción identitaria previa es importante, como la red de relaciones que han creado y mantenido, pero ello no garantiza por sí mismo el éxito en la organización, sino que este es, en parte, producto de un conjunto de acciones más creativas que, teniendo como asiento la tradición, se debaten y discuten dentro de un marco social, político e institucional particular. En este marco son importantes, para el caso tratado, las transformaciones en las dinámicas de distribución del poder en la ciudad a partir de 1997. A fin de cuentas el proceso de organización descrito aquí se desarrolló apenas un año después de que por primera vez fueran elegidos por medio del voto los delegados de las delegaciones de la ciudad, lo que amplió el proceso de democratización de la ciudad y en el que habían quedado al frente de estas demarcaciones geopolíticas muchos candidatos provenientes del partido que se autodefine como de izquierda.

Por ello, pensar este proceso involucra tener en cuenta varios planos, entre los que se encuentra el desarrollo político y el territorial de la ciudad, íntimamente ligados en la etapa actual del desarrollo urbano, porque el conflicto mismo que representa involucra diferentes cuestiones en el marco de la relación de la ciudad con sus pueblos y de la construcción de políticas multi e interculturales. Por lo demás, la experiencia de lucha estudiada aquí también nos muestra la capacidad de organización y movilización de sectores que continuamente encuentran violentadas sus formas de vida.

Finalmente y teniendo en cuenta esto, podríamos pensar en las nuevas amenazas que se ciernen sobre los habitantes de los pueblos. Una organización como la Unión hubiera sido mucho más complicada, y con ello más difícil conseguir sus objetivos, si no hubiera existido aquella organización y vinculación previas, pero ¿existe esto en cuestiones como el agua, los bosques, la defensa de sus cascos urbanos centrales?, ¿deben crearse en caso de no haberlos?, ¿qué figuras serían?

\section{Agradecimientos}

Agradezco los comentarios hechos por el Dr. Hilario Topete, así como de los dictaminadores de la revista Diálogo Andino. 


\section{Referencias Citadas}

Álvarez, L. (coord.)

2011 Pueblos Urbanos. Identidad, Ciudadanía y Territorio en la Ciudad de México. Miguel Ángel Porrúa, CEIICHUNAM, México.

Departamento del Distrito Federal

1984 Reglamento de cementerios del Distrito Federal.

Duhau E. y A. Giglia

2008 Las reglas del Desorden: Habitar la Metrópoli. Siglo XXI, México.

Canto, M.

2004 La disputa teórica sobre las organizaciones civiles. Un asunto político-ideológico. En Las Organizaciones Civiles Mexicanas hoy, editado por J. Cadena, pp. 49-71. UNAMCEIICH, México.

Connolly, P.

2009 Observing the evolution of irregular settlements. Mexico City's colonias populares. International Development Planning Review 31: 1-35.

Escobar, A., S. Álvarez y E. Dagnino. (coords.)

2001 [1999] Política Cultural y Cultura Política. Una Nueva Mirada sobre los Movimientos Sociales Latinoamericanos. Taurus-Instituto Colombiano de Antropología e Historia, Bogotá.

Jasper, J.

2004 A Strategic Approach to Collective Action: Looking for agency in social movement choices. Mobilization: An International Journal 9: 1-16.

Landázuri, G.

2012 La migración, marco de exclusión y discriminación en San Gregorio Atlapulco, México, en Racismos y otras formas de intolerancia de norte a sur en América Latina, coordinado por A. Castellanos y G. Landázuri, pp. 77-98. UAM-Juan Pablos Editor, México.

Luna, M. y J. L. Velasco

2010 Mecanismos de toma de decisiones y desempeño en sistemas asociativos complejos, en Nuevas perspectivas para el estudio de las asociaciones, coordinado por M. Luna y C. Puga, pp. 121-153. Anthropos-UNAM (IIS), México.
Medina, A. (coord.)

2007 La Memoria Negada de la Ciudad de México: sus pueblos Originarios, UNAM-IIA y UACM, México.

Medina, A.

2010 Ciudades españolas, savia india. La trasmutación cultural de las ciudades americanas, en Pueblos, Diásporas y voces de América Latina, coordinado por R. Melgar y R. Cassigoli, pp. 81-101. UNAM, Mexico.

Melucci, A.

1994 ¿Qué hay de nuevo en los "nuevos movimientos sociales"?, en Los nuevos movimientos sociales. De la ideología a la identidad, editado por E. Laraña y J. Gusfield, pp. 119-149. Centro de Investigaciones Sociológicas, Madrid.

Melucci, A.

1996 Challenging codes. Collective action in the information age. Cambridge University Press, UK.

Melucci, A.

1999 Acción colectiva, vida cotidiana y democracia. El Colegio de México, México.

Romero, M. T.

2010 Memoria y defensa de los panteones comunitarios en el Distrito Federal. Nueva Antropología 73: 9-33.

Safa, P.

1998 Vecinos y Vecindarios en la Ciudad de México. Un Estudio sobre la Construcción de las Identidades Vecinales en Coyoacán D.F. Miguel Ángel Porrúa, CIESAS, UAM-I, México.

Snow, D., B. Rochford, S. Worden y R. Benford

2006 Procesos de alineamientos de marcos, micromovilización y participación en movimientos, en $E l$ "análisis de los marcos" en la sociología de los movimientos sociales, coordinado por A. Chihu, pp. 31-82. UAM-I, CONACYT, Miguel Ángel Porrúa, México.

Yanes, P

2007 El desafío de la diversidad. Los pueblos indígenas, la ciudad de México y las políticas del Gobierno del Distrito Federal, 1998-2006, Tesis para optar por el grado de Maestro en Gobierno y Asuntos Públicos, UNAM, Facultad de Ciencias Políticas y Sociales, México.

\section{Notas}

1 La denominación de pueblo originario surge desde los mismos pueblos en encuentros que realizan en los últimos años del siglo pasado y los primeros de este (Medina 2007) para hacer referencia a aquellas comunidades que se reconocen como preexistentes a la constitución de la Ciudad de México (1824) y algunos antes de la conquista española y que mantienen, entre otras cosas, varias tradiciones, familias troncales, un territorio identificable, una relación con su Santo Patrón y ciertos rasgos de la civilización mesoamericana. Este término, sin embargo, es debatido dentro del ámbito académico y en ocasiones se refiere a ellos como pueblos urbanos (Álvarez 2011).

2 Como dice Andrés Medina (2010:95), “en una perspectiva más amplia aparece entonces un complejo sistema de pueblos que tiene como espacio histórico y ecológico el sistema lacustre de la cuenca de México, donde se desarrollan los antiguos estados y las ciudades mesoamericanas, precisamente a la orilla del sistema lacustre".
3 De acuerdo con algunos estudiosos del fenómeno urbano en la ciudad de México, en esta se pueden encontrar seis tipos de poblamiento en la ciudad: ciudad colonial, ciudad central, cabecera conurbada, pueblo no conurbado, colonia popular, conjunto habitacional, residencial medio y residencial alto (Duhau y Giglia 2008; Connolly 2009).

4 En este trabajo se entiende la idea de comunidad referida más a los vínculos entre individuos que se reconocen como parte de algún conjunto más amplio y definen, entre otras cosas, sus formas de pertenencia y exclusión. Evoca entonces un proyecto en constante construcción y es pensado como un conjunto de relaciones, no como una entidad en sí. Además, términos como localidad hacen referencia únicamente a las cuestiones más formales de aspectos como su ubicación, características sociodemográficas y económicas, entre otros.

5 Durante la gestión de Marcelo Ebrard (2006-2012) se realizaron dotaciones de tierras para distintos cementerios, en ocasiones mediante expropiaciones. Aquí no se incluye esa 
superficie de tierra porque en algunos casos, como sucedió por ejemplo en Culhuacán, en la delegación Iztapalapa, este proceso no ha terminado, pues los afectados por esas expropiaciones han interpuesto recursos para que ello no se lleve a cabo. Por lo demás, las dotaciones del gobierno se han enfocado en ampliar los terrenos de los cementerios ya existentes.

6 En 1997 el estatus jurídico de Ciudad de México pasó de "Departamento" a "Gobierno", pero este cambio no parece haber afectado las disposiciones establecidas en este reglamento.

7 Este tema es muy amplio y no es la razón de este trabajo hablar de ello con más detalle. Pero es necesario decir que, si bien esta diferenciación entre los dos "sujetos" no siempre es un modelo que impide la unión entre ellos, de tal forma que en los sistemas de cargos, como las mayordomías, es posible encontrar avecindados participando, conlleva un alto riesgo de exclusión, como se han descrito en diferentes investigaciones (ver: Landázuri 2012).

8 Esta fue la primera de tres propuestas que se presentaron entre los años 2001 y 2004. Debido a que este trabajo se centró en los aspectos iniciales de la organización, no se hablará de lo que sucedió con aquellas otras propuestas, baste decir que entre esos años la organización tuvo periodos de menor actividad mientras no había "amenazas", volviendo a actividades más continuas cuando se enteraron de estas otras propuestas, que también lograron echar abajo.

9 Mediante una Ley de Hacienda del Departamento del Distrito Federal se cancela el régimen de perpetuidades so argumento de un "alto índice de abandono y el agotamiento de espacios disponibles para inhumar" (Romero 2010). Además, el Reglamento de Cementerios del Distrito Federal establece cuatro temporalidades para las fosas: "La temporalidad mínima confiere el derecho de uso sobre una fosa durante siete años (...) La temporalidad máxima confiere el derecho de uso sobre una fosa durante un plazo de siete años, refrenable por dos períodos iguales (...) La temporalidad prorrogable confiere el derecho de uso sobre una cripta familiar o un nicho durante siete años, contados a partir de la fecha de celebración del convenio y refrendable cada siete años por tiempo indefinido (...) La temporalidad indefinida confiere el derecho de uso sobre un nicho por tiempo indeterminado" (Departamento 1984).

10 Entrevista al Sr. Hilario Salvador, integrante del patronato del panteón en el pueblo de San Francisco Culhuacán, realizada en la Ciudad de México en el 2011.

11 Para Alberto Melucci (1996), por ejemplo, el dinero no es el único tipo de recurso con el que una organización o movimiento cuenta, también pueden pensarse el apoyo y el consenso como recursos utilizables.

12 El término micromovilización es parte del entramado conceptual de las teorías anglosajonas de los movimientos sociales, preocupadas especialmente por las estrategias que despliegan los movimientos en un entorno político particular. Pero es posible vincularlo también a cuestiones más culturales, como el esfuerzo que David Snow y otros hicieron por integrar este concepto dentro del proceso de creación de marcos cognitivos que buscan vincular los intereses de la organización con los de los individuos que participan en ellos. Ver: Snow et al. (2006).

13 La figura del Coordinador de Enlace Territorial existe en cuatro delegaciones de la Ciudad de México: Tláhuac, Milpa Alta, Xochimilco y Tlalpan. Refiere a una persona elegida por la comunidad y designada como el responsable de la comunicación entre esta y las autoridades delegacionales. También está facultado para dar solución a ciertas problemáticas al interior de las comunidades. Sin embargo, es una figura ambigua jurídicamente, en lo principal porque a pesar de haber sido elegido por la comunidad, no tiene una inserción clara en la estructura de gobierno de las delegaciones.

14 Entrevista al Sr. Víctor Martínez Flores, originario del pueblo de Los Reyes Coyoacán e integrante de diversos cargos en la comunidad, entre ellos las mayordomías y la comisión del panteón, realizada en la Ciudad de México en el 2011.

15 Una de las razones de esta falta de la autodefinición como "originarios" puede encontrarse en las fechas en las que esta Unión se conforma, a inicios del siglo XXI. En estos años tenía poco tiempo de que se había comenzado a usar el término "pueblos originarios" para referirse a estas comunidades, aunque rápidamente tomaba fuerza (Medina 2007).

16 Entrevista al Sr. Felipe Cruz Hernández, originario del pueblo de La Magdalena Contreras, realizada en la Ciudad de México en el 2011.

17 De acuerdo con Teresa Romero (2010), en Iztacalco sí existen panteones vecinales. Además, en Benito Juárez también parece haber por lo menos uno, el de Xoco, aunque no esté bajo la administración de los habitantes del pueblo.

18 Entrevista a Víctor Martínez Flores.

19 Entrevista a Víctor Martínez Flores. Para una descripción de distintas etapas en la conformación de la colonia y de su relación con el pueblo de Los Reyes ver: Safa (1998).

20 Para un mayor detalle sobre esta y las siguientes marchas ver: Romero (2010): "el periódico El Universal (5 de abril de 2002) se trató de 200 personas, mientras La Jornada de ese mismo día reporta cerca de mil manifestantes. Luego de cuatro horas de plantón frente a la Asamblea Legislativa, durante el cual se obstruyeron las puertas de acceso, la comisión negociadora consigue la firma de un acuerdo en el que diputados de los diferentes partidos se comprometen a no aprobar la iniciativa de ley [...] (La Prensa, 8 de abril de 2002: 18)".

21 Entrevista a Hilario Salvador.

22 En palabras del entrevistado Víctor Martínez, la mayordomía se constituye como A.C. "fundamentalmente para la defensa", pues había una situación de robo de piezas arqueológicas por parte de la Iglesia, para poder levantar una demanda se les requería constituirse en una figura legal, que les exigía el gobierno. La organización de esta AC es bastante compleja. 
